\title{
On a class of quartic Diophantine equations
}

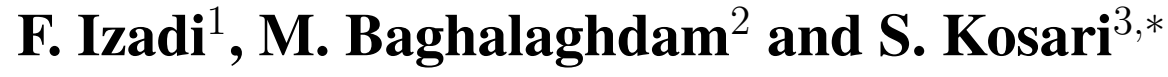 \\ ${ }^{1}$ Department of Mathematics, Faculty of Science, Urmia University \\ Urmia 165-57153, Iran \\ e-mail: f.izadi@urmia.ac.ir \\ ${ }^{2}$ Department of Mathematics, Faculty of Science, Azarbaijan Shahid Madani University \\ Tabriz 53751-71379, Iran \\ e-mail: mehdi.baghalaghdameyahoo.com \\ ${ }^{3}$ Institute of Computing Science and Technology, Guangzhou University \\ Guangzhou, 510006, China \\ e-mail: saeedkosari38@gzhu.edu.cn \\ * Corresponding author
}

Received: 22 January 2020 Revised: 27 November $2020 \quad$ Accepted:29 November 2020

\begin{abstract}
In this paper, by using elliptic curves theory, we study the quartic Diophantine equation (DE) $\sum_{i=1}^{n} a_{i} x_{i}^{4}=\sum_{j=1}^{n} a_{j} y_{j}^{4}$, where $a_{i}$ and $n \geq 3$ are fixed arbitrary integers. We try to transform this quartic to a cubic elliptic curve of positive rank. We solve the equation for some values of $a_{i}$ and $n=3,4$, and find infinitely many nontrivial solutions for each case in natural numbers, and show among other things, how some numbers can be written as sums of three, four, or more biquadrates in two different ways. While our method can be used for solving the equation for $n \geq 3$, this paper will be restricted to the examples where $n=3,4$. Finally, we explain how to solve more general cases ( $n \geq 4$ ) without giving concrete examples to case $n \geq 5$.
\end{abstract}

Keywords: Quartic Diophantine equations, Biquadrates, Elliptic curves.

2010 Mathematics Classification: 11D45, 11D72, 11D25, 11G05, 14H52.

\section{Introduction}

Quartic Diophantine equations has been studied by many authors. Euler conjectured that the DE $A^{4}+B^{4}+C^{4}=D^{4}$, or more generally $A_{1}^{N}+A_{2}^{N}+\cdots+A_{N-1}^{N}=A_{N}^{N},(N \geq 4)$, has no solution in positive integers (see [3]). Nearly two centuries later, a computer search (see [6]) found the first counterexample to the general conjecture (for $N=5$ ): $27^{5}+84^{5}+110^{5}+133^{5}=144^{5}$. 
In 1986, Noam Elkies, by using elliptic curves, found an infinite series of counterexamples for the $K=4$ case (see [4]). His smallest counterexample was:

$$
2682440^{4}+15365639^{4}+18796760^{4}=20615673^{4} .
$$

In [1] Bremner, Choudhry, and Ulas constructed several infinite families of diagonal quartic surfaces $a x^{4}+b y^{4}+c z^{4}+d w^{4}=0$ (where $a, b, c, d$ are non-zero integers) with infinitely many rational points and satisfying the condition $a b c d$ is not a square.

In [2], we studied the DE $A^{4}+h B^{4}=C^{4}+h D^{4}$ and express two different methods for solvig it.

In this paper, we are interested in the study of the DE:

$$
\sum_{i=1}^{n} a_{i} x_{i}^{4}=\sum_{j=1}^{n} a_{j} y_{j}^{4}
$$

where $a_{i}, n \geq 3$ are fixed arbitrary integers. To the best of our knowledge the DE (1) has not already been considered by any other authors.

We prove the following two theorems:

Theorem 1. Consider the DE

$$
a x^{4}+b y^{4}+c z^{4}=a u^{4}+b v^{4}+c w^{4}
$$

where all the coefficients are fixed arbitrary integers. There exists a corresponding elliptic curve of the form $Y^{2}=X^{3}+f X^{2}+g X+h$, in which the coefficients $f, g$, and h are all functions of a, $b, c$ and two other rational parameters $A$ and $B$, to be found later. If the elliptic curve has positive rank, depending on the values of $A$ and $B$, the DE(2) has infinitely many integer solutions.

Proof. Let $x=m+p, y=m-q, z=m-s, u=m-p, v=m+q$ and $w=m+s$, where all variables are rational numbers. By substituting these variables in the DE (2), and after some simplification, we obtain:

$$
m^{2}(a p-b q-c s)=-a p^{3}+b q^{3}+c s^{3}
$$

Let $a p-b q-c s=1$ and $m^{2}=-a p^{3}+b q^{3}+c s^{3}$. Also let us take $q=A s+B$, where $A, B \in \mathbb{Q}$.

By substituting $q=A s+B$ and $p=\frac{b}{a} q+\frac{c}{a} s+\frac{1}{a}$, into the equation $m^{2}=-a p^{3}+b q^{3}+c s^{3}$, and after some simplification, we arrive at the cubic elliptic curve:

$$
\begin{aligned}
m^{2}= & \left(-a\left(\frac{b A+c}{a}\right)^{3}+b A^{3}+c\right) s^{3}+\left(-3 a\left(\frac{b A+c}{a}\right)^{2} \cdot\left(\frac{b B+1}{a}\right)+3 A^{2} B b\right) s^{2} \\
& +\left(-3 a\left(\frac{b A+c}{a}\right) \cdot\left(\frac{b B+1}{a}\right)^{2}+3 A B^{2} b\right) s+\left(-a\left(\frac{b B+1}{a}\right)^{3}+b B^{3}\right) .
\end{aligned}
$$

By multiplying both sides of this elliptic curve by $\left(-a\left(\frac{b A+c}{a}\right)^{3}+b A^{3}+c\right)^{2}$, and letting

$$
Y=\left(-a\left(\frac{b A+c}{a}\right)^{3}+b A^{3}+c\right) m
$$


and

$$
X=\left(-a\left(\frac{b A+c}{a}\right)^{3}+b A^{3}+c\right) s,
$$

we get the new elliptic curve

$$
Y^{2}=X^{3}+f X^{2}+g X+h
$$

where

$$
\begin{aligned}
& f=\left(-3 a\left(\frac{b A+c}{a}\right)^{2} \cdot\left(\frac{b B+1}{a}\right)+3 A^{2} B b\right), \\
& g=\left(-3 a\left(\frac{b A+c}{a}\right) \cdot\left(\frac{b B+1}{a}\right)^{2}+3 A B^{2} b\right) \cdot\left(-a\left(\frac{b A+c}{a}\right)^{3}+b A^{3}+c\right), \\
& h=\left(-a\left(\frac{b B+1}{a}\right)^{3}+b B^{3}\right) \cdot\left(-a\left(\frac{b A+c}{a}\right)^{3}+b A^{3}+c\right)^{2} .
\end{aligned}
$$

Then if the above elliptic curve has positive rank (for every $a, b, c$, this is done by choosing appropriate values for $A$ and $B$ ), by calculating $m, s, q, p, m \pm p, m \pm q, m \pm s$, from the relations (3), (4), $q=A s+B$ and $p=\frac{b}{a} q+\frac{c}{a} s+\frac{1}{a}$, after some simplification and canceling the denominators of $m \pm p, m \pm q, m \pm s$, we may obtain infinitely many integer solutions for the DE (2). Now the proof is completed.

Now we are going to work out some examples.

Example 1. $x^{4}+2 y^{4}+3 z^{4}=u^{4}+2 v^{4}+3 w^{4}$ we may assume that $A=4, B=0, a=1, b=2$, $c=3$. (For every arbitrary $a, b, c$, we may choose the other appropriate values for $A$ and $B$ so that the rank of the elliptic curve (5) to be $\geq 1$.)

Then the corresponding cubic elliptic curve is:

$$
Y^{2}=X^{3}-363 X^{2}+39600 X-1200^{2} .
$$

This has rank 1 and generator $P=(X, Y)=\left(\frac{3625}{16}, \frac{46525}{64}\right)$, giving the solution

$$
5169^{4}+2 \cdot(459)^{4}+3 \cdot(1281)^{4}=1447^{4}+2 \cdot(4181)^{4}+3 \cdot(2441)^{4},
$$

to $(2)$

Example 2. We now express the sum of three biquadrates in two different ways. Let us this time take $A=-10, B=0, a=b=c=1$. Then the elliptic curve (5) becomes

$$
Y^{2}=X^{3}-243 X^{2}-7290 X-270^{2},
$$

which has rank 1 and generator $P=(X, Y)=(450,6210)$.

The points $P, 2 P=\left(X^{\prime}, Y^{\prime}\right)=\left(\frac{606357}{2116}, \frac{-115780401}{97336}\right)$ and $3 P=\left(X^{\prime \prime}, Y^{\prime \prime}\right)=\left(\frac{255306774610}{164070481}\right.$, $\left.\frac{-118288360159623370}{2101578791129}\right)$ yield the solutions:

$$
\begin{aligned}
19^{4}+74^{4}+117^{4} & =21^{4}+64^{4}+119^{4}, \\
17948013^{4}+43856069^{4}+9765331^{4} & =43676991^{4}+18127091^{4}+15963647^{4},
\end{aligned}
$$

and

$$
\begin{aligned}
& 8828891360220313^{4}+15099060491941827^{4}+11501813568364388^{4} \\
& =14828780671704361^{4}+8558611539982847^{4}+12155858463560286^{4} .
\end{aligned}
$$


By changing the value of $A$, and taking $A=-11, B=0$, we get the new elliptic curve $Y^{2}=X^{3}-300 X^{2}-9900 X-108900$, with rank 1 and generator $P=(X, Y)=\left(\frac{7241401}{11664}\right.$, $\left.\frac{13649431501}{1259712}\right)$, giving the solution

$$
\begin{aligned}
& 5413013461^{4}+22252215889^{4}+12867360193^{4} \\
& =21885849541^{4}+5046647113^{4}+14431502809^{4} .
\end{aligned}
$$

Example 3. $A^{4}+h B^{4}=C^{4}+h D^{4}$.

If in the elliptic curve (5) we take $c=0, a \neq \pm 1$, (If $a= \pm 1$, we do not get an elliptic curve, this means that $g=h=0$.), we can solve the $D E$ 's of the form $a x^{4}+b y^{4}=a u^{4}+b v^{4}$.

As an example, if we take $A=2, B=0, a=103, b=1, c=0$, we get the elliptic curve $Y^{2}=X^{3}-\frac{12}{10609} X^{2}-\frac{509184}{112550881} X-\frac{7201898496}{1194052296529}$, with rank 1 and generator $P=(X, Y)=$ $\left(\frac{8148673}{22448644}, \frac{21273981079}{106361675272}\right)$, giving the solution:

$$
4303295713^{4}+103 .(2329537023)^{4}=7576215879^{4}+103 .(943383143)^{4} .
$$

Theorem 2. Consider the DE (1), where $n \geq 4$, and all the coefficients are fixed arbitrary integers. There exists a corresponding elliptic curve of the form $Y^{2}=X^{3}+f X^{2}+g X+h$, in which the coefficients $f, g$, and h are all functions of $a_{i}$ and the other rational parameters $A_{i}, B_{i}$, to be found later. If the elliptic curve has positive rank, depending on the values of $A_{i}, B_{i}$, the DE (1) has infinitely many integer solutions.

Proof. Let $x_{i}=m+p_{i}$ and $y_{i}=m-p_{i},($ For $1 \leq i \leq n)$, where $m, p_{i} \in \mathbb{Q}$. After substituting these variables in the DE (1), we get:

$$
m^{2}\left(a_{1} p_{1}+\cdots+a_{n} p_{n}\right)=-a_{1} p_{1}^{3}-\cdots-a_{n} p_{n}^{3} .
$$

Set $\left(a_{1} p_{1}+\cdots+a_{n} p_{n}\right)=1$, and $m^{2}=-a_{1} p_{1}^{3}-\cdots-a_{n} p_{n}^{3}$.

Also let us take $p_{i}=A_{i} p_{n}+B_{i}$ (for $2 \leq i \leq n-1$ ).

By substituting $p_{1}=-\frac{a_{2}}{a_{1}} p_{2}-\cdots-\frac{a_{n-1}}{a_{1}} p_{n-1}-\frac{a_{n}}{a_{1}} p_{n}+\frac{1}{a_{1}}=G p_{n}+H$, where

$$
G=\frac{-a_{2} A_{2}-a_{3} A_{3}-\cdots-a_{n-1} A_{n-1}-a_{n}}{a_{1}},
$$

and

$$
H=\frac{-a_{2} B_{2}-a_{3} B_{3}-\cdots-a_{n-1} B_{n-1}+1}{a_{1}},
$$

and $p_{i}=A_{i} p_{n}+B_{i}$ (for $2 \leq i \leq n-1$ ), into the Eq. $m^{2}=-a_{1} p_{1}^{3}-\cdots-a_{n} p_{n}^{3}$, and after some simplification, we obtain the elliptic curve:

$$
m^{2}=L_{1} p_{n}^{3}+L_{2} p_{n}^{2}+L_{3} p_{n}+L_{4},
$$

where

$$
\begin{aligned}
& L_{1}=\left(-a_{1} G^{3}-a_{2} A_{2}^{3}-a_{3} A_{3}^{3}-\cdots-a_{n-1} A_{n-1}^{3}-a_{n}\right), \\
& L_{2}=\left(-3 a_{1} G^{2} H-3 a_{2} A_{2}^{2} B_{2}-3 a_{3} A_{3}^{2} B_{3}-\cdots-3 a_{n-1} A_{n-1}^{2} B_{n-1}\right), \\
& L_{3}=\left(-3 a_{1} G H^{2}-3 a_{2} A_{2} B_{2}^{2}-3 a_{3} A_{3} B_{3}^{2}-\cdots-3 a_{n-1} A_{n-1} B_{n-1}^{2}\right), \\
& L_{4}=\left(-a_{1} H^{3}-a_{2} B_{2}^{3}-a_{3} B_{3}^{3}-\cdots-a_{n-1} B_{n-1}^{3}\right) .
\end{aligned}
$$


By multiplying both sides of this elliptic curve by

$$
L_{1}^{2}=\left(-a_{1} G^{3}-a_{2} A_{2}^{3}-a_{3} A_{3}^{3}-\cdots-a_{n-1} A_{n-1}^{3}-a_{n}\right)^{2}
$$

and letting

$$
Y=\left(-a_{1} G^{3}-a_{2} A_{2}^{3}-a_{3} A_{3}^{3}-\cdots-a_{n-1} A_{n-1}^{3}-a_{n}\right) m
$$

and

$$
X=\left(-a_{1} G^{3}-a_{2} A_{2}^{3}-a_{3} A_{3}^{3}-\cdots-a_{n-1} A_{n-1}^{3}-a_{n}\right) p_{n},
$$

we get the new elliptic curve $Y^{2}=X^{3}+f X^{2}+g X+h$, where

$$
\begin{aligned}
f= & \left(-3 a_{1} G^{2} H-3 a_{2} A_{2}^{2} B_{2}-3 a_{3} A_{3}^{2} B_{3}-\cdots-3 a_{n-1} A_{n-1}^{2} B_{n-1}\right), \\
g= & \left(-3 a_{1} G H^{2}-3 a_{2} A_{2} B_{2}^{2}-3 a_{3} A_{3} B_{3}^{2}-\cdots-3 a_{n-1} A_{n-1} B_{n-1}^{2}\right) \\
& \left(-a_{1} G^{3}-a_{2} A_{2}^{3}-a_{3} A_{3}^{3}-\cdots-a_{n-1} A_{n-1}^{3}-a_{n}\right), \\
h= & \left(-a_{1} H^{3}-a_{2} B_{2}^{3}-a_{3} B_{3}^{3}-\cdots-a_{n-1} B_{n-1}^{3}\right) \\
& \left(-a_{1} G^{3}-a_{2} A_{2}^{3}-a_{3} A_{3}^{3}-\cdots-a_{n-1} A_{n-1}^{3}-a_{n}\right)^{2} .
\end{aligned}
$$

Then if the above elliptic curve has positive rank (For every arbitrary integers $a_{i}$, this is done by choosing appropriate values for $A_{i}$ and $B_{i}$ ), by calculating $m, p_{i}, m \pm p_{i}$, from the relations (7), (8) and $p_{i}=A_{i} p_{n}+B_{i}$ (for $2 \leq i \leq n-1$ ), $p_{1}=G p_{n}+H$, after some simplification and canceling the denominators of $m \pm p_{i}$, we may obtain infinitely many integer solutions for the DE (1). Now the proof is completed.

Example 4. $x^{4}+1000 y^{4}+1000 z^{4}+1000 t^{4}=u^{4}+1000 v^{4}+1000 w^{4}+1000 h^{4}$.

By letting $x=m+p, y=m-q, z=m-s, t=m+r, u=m-p, v=m+q, w=m+s$, $h=m-r, p-1000 q-1000 s+1000 r=1, m^{2}=-p^{3}+1000 q^{3}+1000 s^{3}-1000 r^{3}, q=2 s$ and $r=3 s$, we get the corresponding elliptic curve $Y^{2}=X^{3}-18000^{2}$ which has rank 1 and generator $P=(X, Y)=(1000,26000)$, giving the solution

$$
8^{4}+1000 \cdot(24)^{4}+1000 \cdot(25)^{4}+1000 \cdot(29)^{4}=44^{4}+1000 \cdot(23)^{4}+1000 \cdot(27)^{4}+1000 \cdot(28)^{4} .
$$

It is interesting to note that $24+25+29=23+27+28$.

Example 5. Let us show how to write the sums of four biquadrates in two different ways. As the above example, by taking $q=3 s, r=7 s, p-q-s+r=1$ and $m^{2}=-p^{3}+q^{3}+s^{3}-r^{3}$, the corresponding elliptic curve becomes $Y^{2}=X^{3}-27 X^{2}-2592 X-288^{2}$. This has rank 1 and generator $P=(X, Y)=(328,5608)$. The points $P, 2 P=\left(X^{\prime}, Y^{\prime}\right)=\left(\frac{48232180}{491401}, \frac{203244176836}{344472101}\right)$, and $3 P=\left(X^{\prime \prime}, Y^{\prime \prime}\right)=\left(\frac{61771836632004160}{797318963764569}, \frac{-3119953836085429109330528}{22513765616829041228253}\right)$ yield the solutions:

$271^{4}+289^{4}+330^{4}+494^{4}=207^{4}+371^{4}+412^{4}+430^{4}$,

$325492151^{4}+12726487787^{4}+21179177332^{4}+54989935512^{4}$

$=50485552058^{4}+38084556422^{4}+29631866877^{4}+4178891303^{4}$,

and 
$463645068132430760337286^{4}+261021177580969389283009^{4}$

$+152006097445436236205389^{4}+284054223096696376105091^{4}$

$=268647953377091441004128^{4}+66024062825630069949851^{4}$

$+42991017309903083127769^{4}+479051337852035695438249^{4}$.

By taking $q=7 s$ and $r=4 s$, we get the new elliptic curve $Y^{2}=X^{3}-48 X^{2}-2592 X-46656$, with rank 1 and generator $P=(X, Y)=\left(\frac{4188}{49}, \frac{25488}{343}\right)$, giving the solution:

$$
18070^{4}+14977^{4}+319^{4}+11896^{4}=13822^{4}+19225^{4}+4567^{4}+7648^{4} .
$$

Remark. By choosing further points on the above elliptic curves such as $4 P, 5 P, \ldots$, we obtain infinitely many solutions for each case of the above Diophantine equations.

The Sage software has been used for calculating the rank of the elliptic curves (see [6]).

\section{Acknowledgements}

We are very grateful to the referee for the careful reading of the paper and giving several useful comments which improved the quality of the paper.

\section{References}

[1] Bremner, A., Choudhry, A., \& Ulas, M. (2014). Constructions of diagonal quartic and sextic surfaces with infinitely many rational points, International Journal of Number Theory, 10(7), 1675-1698.

[2] Baghalaghdam, M., \& Izadi, F. Is the quartic Diophantine equation $A^{4}+h B^{4}=C^{4}+h D^{4}$, solvable for any integer $h$ ?, submitted.

[3] Dickson, L. E. (1934). History of the Theory of Numbers, Vol. II: Diophantine Analysis, G. E. Stechert Co., New York.

[4] Elkies, N. (1988). On $A^{4}+B^{4}+C^{4}=D^{4}$. Mathematics of Computation, 51(184), 825-835.

[5] Lander, L. J., \& Parkin, T. R. (1966). Counterexamples to Euler's conjecture on sums of like powers, Bull. Amer. Math. Soc, 72, 1079.

[6] Sage software, available from http://sagemath.org. 